\title{
Bioanalysis
}

\section{Sample extraction techniques for biological samples: recent advances and novel applications}

“...good sample preparation can serve as a way to reduce the complexity of biological samples by selective extraction of the preferred analytes or as a way to enrich these analytes in a new medium"

Keywords: biological matrices • extraction • sample preparation

Sample preparation is an important part of modern analytical chemistry and is performed to ensure high reliability, sensitivity, selectivity and reproducibility of an analytical method. Even though recent advances in analytical instrumentation have introduced systems that to a large degree can give good quality results from samples without the need of pretreatment, there will always be a need for effective sample preparation to further improve the analytical performance. In this area, good sample preparation can serve as a way to reduce the complexity of biological samples by selective extraction of the preferred analytes or as a way to enrich these analytes in a new medium. The reduced complexity of a sample matrix will be beneficial for both the sensitivity and selectivity of the method. Additionally, the reduced amount of interfering compounds can improve the robustness of the method and reduce the degree of instrument contamination and thus the need for maintenance. Enrichment of analytes in a new medium can both serve as a way to increase sensitivity and ensure compatibility with an analytical instrument. According to these factors, an inefficient sample preparation can easily become a bottle-neck for the quality of an analytical method.

Especially in analysis of biological samples, there is often a need to detect low abundant analytes in complex matrices. Examples of this are biomarkers for different diseases, drugs with a low therapeutic concentration and their metabolites, or endogenous peptides and proteins that are present in biological fluids in a low concentration. In these instances, proper sample preparation is often necessary to ensure a high enrichment of analytes to reach the detection limit, and as a way to remove matrix components that otherwise might interfere with the detection of the target analytes. Some biological sample matrices can for instance introduce severe matrix effects, which can lead to signal enhancement or suppression in the detector, and it is preferable that these effects are kept to a minimum to obtain reproducible results.

The need for efficient sample cleanup with minimum time consumption has been an important factor in modern sample preparation techniques. In this case, automated systems, taking advantage of miniaturized and hyphenated techniques, have been a popular way to reduce total time consumption and labor involved in the analytical method. These criteria, in addition to a focus on minimizing the use of hazardous chemical compounds for safety and environmental reasons, have become increasingly popular for novel sample preparation techniques and applications.

This special issue gives an overview of recent advances and novel applications in extraction techniques for biological samples and includes extractions into solid media, extractions into liquid media, as well as other techniques. Common for all the techniques described are that they represent innovative strategies toward solving today's challenges with sample preparation of biological samples.
Astrid Gjelstad

School of Pharmacy, University of Oslo, Norway

\section{Knut Fredrik Seip}

Author for correspondence:

School of Pharmacy, University of Oslo, Norway

k.f.seip@farmasi.uio.no 


\section{Foreword Gjelstad \& Seip}

\section{Financial \& competing interests disclosure}

The authors have no relevant affiliations or financial involvement with any organization or entity with a financial interest in or financial conflict with the subject matter or materials discussed in the manuscript. This includes employ- ment, consultancies, honoraria, stock ownership or options, expert testimony, grants or patents received or pending, or royalties.

No writing assistance was utilized in the production of this manuscript. 